\title{
Metabolomics Comparison Between Ovine and Bovine Serum at Mid-lactation
}

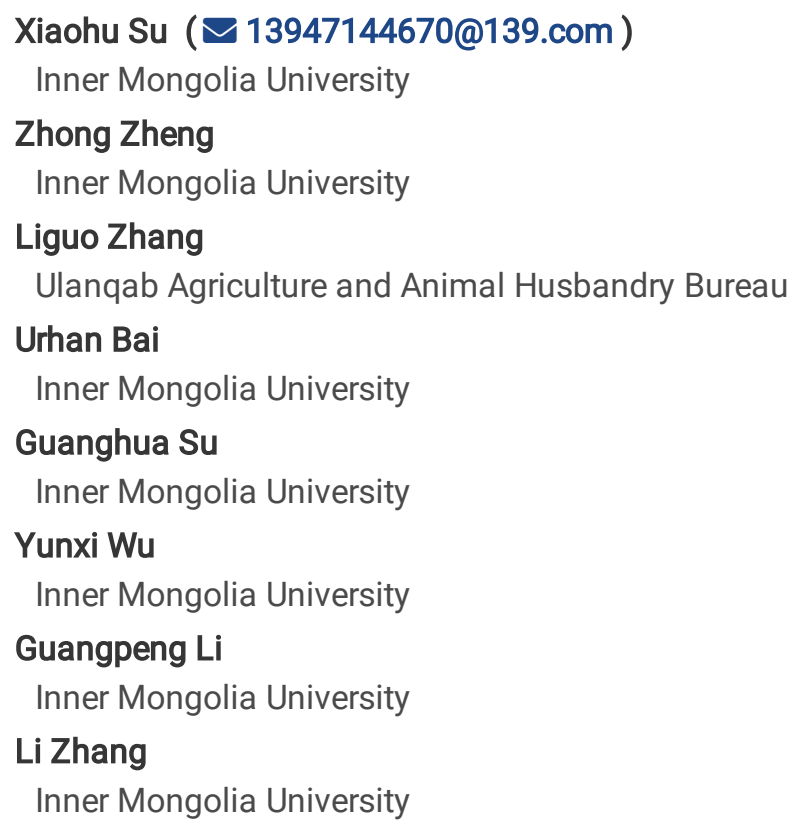




\section{Abstract}

The ovine milk owns higher lactoprotein, fat and other solids than bovine milk. However, the mechanism was not fully clear. To discover the specific mechanism, an untargeted metabolomics analyze of serum at mid-lactation by liquid chromagraphy-mass spectrometry (LC-MS) was performed. Then multivariate statistical analysis was carried out to find the specific differences. Final the different abundant metabolites were functionally enrichment by KEGG. In total, 1615 metabolites were detected in serum and 486 were annotated. The the largest metabolic category was lipids and lipid-like molecules (188 metabolites). 412 metabolites were identified as differential metabolites between two groups. KEGG pathway enrichment showed that 18 and 10 functional pathways of differential metabolites were enriched at positive and negative ion mode, separately. Notably, hernandezine, which is a novel AMPK activator, may play a role in the formation of lactoprotein of ovine milk. The results indicated that there may be different biological effects between two species serum. The serum metabolites could make help for the formation of milk.

\section{Introduction}

Milk is a nutritious food which contains multiple components such as proteins, fat, lactose, bioactive peptides and micronutrients ${ }^{1}$. Although the bovine milk occupies the major marker, small ruminants' milk, such as caprine and ovine, are emphasized nowadays. Compared with bovine milk, the ovine milk owns higher percentages of lactoprotein, milk fat and so on ${ }^{2}$. The molecular composition of milk is influenced by various genetic and environmental factors. However, the specific regulation mechanism of milk composition differences is unclear.

Metabolites are effectively the end products of complex interactions occurring inside the cell (the genome) and outside the cell or organism (the environment). The advanced analytical chemistry techniques were used to comprehensively measure large numbers of small molecule metabolites in cells, tissues and biofluids, named metabolomics ${ }^{3}$. The blood metabolomics study was largely used to explain or identify the economic traits of livestock ${ }^{4-6}$. For a part of milk metabolites were from blood and the blood metabolites could regulate the biological function of mammary cells, the serum metabolomics would be one of useful strategy to explain the mechanism of milk composition differences between different species ${ }^{7-9}$. In dairy cows, Hippuric acid, nicotinamide and pelargonic acid of serum could be milk protein biomarkers ${ }^{10}$. However, little research to discover milk traits differences between species through blood metabolomics. One cause may be that the metabolomics is affected by various factors, such as the genome and the environment, and it is hard to compare at ideal research conditions.

In this study, we fed the ewes and cows at same place to maximize eliminate the impact of environment. We analyzed the milk composition and the blood serum metabolome of ovine and bovine at mid-lactation. The aim of this study was to partly explain the mechanism of milk composition differences between two species through serum metabolomics analysis. It would be helpful for dairy stock's feeding and understanding of milk composition formation basis.

\section{Materials And Methods}

\section{Ethical statement}

Animal manipulations in this study including welfare, husbandry and experimental sampling were approved by the Animal Ethics Committee of Inner Mongolia University (Permit number: IMU-IACUC-2018-B78C). All procedures involving animals were approved by the Ethical Principles for the Use of Animals for Scientific Purposes of the Inner Mongolia University of China. All experiments were performed according to Chinese laws and institutional guidelines.

\section{Animals and samples preparation}

The breeds of this research were Holstein cow and F1 cross-breed from Small-Tailed Han and DairyMeade sheep. All were 2 4 years and parous. The animals which were used for this study were fed at the standard conditions and the same region of Mengtianran Dairy Co. Ltd. (Ulanqab, Inner Mongolia autonomous region, China). The blood samples were collected from similar physical individuals and 6 of each group. The time of samples collection was D90 after parturition. Blood samples were collected in vacuum blood collection tubes. Then, the samples were centrifuged at $3000 \times \mathrm{g} 4{ }^{\circ} \mathrm{C}$ for $15 \mathrm{~min}$ to obtain the corresponding serum within 30 min of collection. All samples were stored in liquid nitrogen until analysis. 


\section{LC-MS analysis conditions}

The LC-MS/MS analysis was processed by Novogene Co. LTD. (Beijing, China). The detailed processes of metabolies annotation and identification analysis were followed as Wang et al. ${ }^{11}$

Metabolites Extraction

The samples $(100 \mu \mathrm{L})$ and prechilled methanol $(400 \mu \mathrm{L})$ were mixed by well vortexing. The samples were incubated on ice for 5 $\mathrm{min}$ and then were centrifuged at $15000 \mathrm{rpm}, 4^{\circ} \mathrm{C}$ for $5 \mathrm{~min}$. A some of supernatant was diluted to final concentration containing $60 \%$ methanol by LC-MS grade water. The samples were subsequently transferred to a fresh Eppendorf tube with $0.22 \mu \mathrm{m}$ filter and then were centrifuged at $15000 \mathrm{~g}, 4^{\circ} \mathrm{C}$ for $10 \mathrm{~min}$. Finally, the filtrate was injected into the LC-MS/MS system analysis.

UHPLC-MS/MS Analysis

LC-MS/MS analyses were performed using a Vanquish UHPLC system (Thermo Fisher) coupled with an Orbitrap Q Exactive series mass spectrometer (Thermo Fisher). Samples were injected onto an Hyperil Gold column $(100 \times 2.1 \mathrm{~mm}, 1.9 \mu \mathrm{m})$ using a 16-min linear gradient at a flow rate of $0.2 \mathrm{~mL} / \mathrm{min}$. The eluents for the positive polarity mode were eluent $\mathrm{A}(0.1 \% \mathrm{FA}$ in Water $)$ and eluent $B$ (Methanol). The eluents for the negative polarity mode were eluent $A(5 \mathrm{mM}$ ammonium acetate, $\mathrm{pH} 9.0$ ) and eluent $\mathrm{B}$ (Methanol).The solvent gradient was set as follows: 2\% B, $1.5 \mathrm{~min} ; 2-100 \% \mathrm{~B}, 12.0 \mathrm{~min} ; 100 \% \mathrm{~B}, 14.0 \mathrm{~min} \varangle 100-2 \% \mathrm{~B}, 14.1 \mathrm{~min} \varangle 2 \%$ $\mathrm{B}, 16 \mathrm{~min}$. Q Exactive mass series spectrometer was operated in positive/negative polarity mode with spray voltage of $3.2 \mathrm{kV}$, capillary temperature of $320^{\circ} \mathrm{C}$, sheath gas flow rate of 35 arb and aux gasflow rate of 10 arb.

\section{Metabolomics data processing}

Database search

The raw data files generated by UHPLC-MS/MS were processed using the Compound Discoverer 3.1 (CD3.1, Thermo Fisher) to perform peak alignment, peak picking, and quantitation for each metabolite. The normalized data was used to predict the molecular formula based on additive ions, molecular ion peaks and fragment ions. And then peaks were matched with the mzCloud (https://www.mzcloud.org/) and ChemSpider (http://www.chemspider.com/) database to obtained the accurate qualitative and relative quantitative results. Statistical analyses were performed using the statistical software $R(R$ version $R-3.4 .3)$, Python (Python 2.7.6 version) and CentOS (CentOS release 6.6), When data were not normally distributed, normal transformations were attempted using of area normalization method.

Data Analysis

These metabolites were annotated using the HMDB database ( http://www.hmdb.ca/). Principal components analysis (PCA) and Partial least squares discriminant analysis (PLS-DA) were performed at metaX (a flexible and comprehensive software for processing metabolomics data). Volcano plots were used to filter metabolites of interest which based on Log2 (FC) and -log10 (Pvalue) of metabolites.

The functions of these metabolites and metabolic pathways were studied using the KEGG database. The metabolic pathway enrichment of differential metabolites were performed, when ratio were satisfied by $x / n>y / N$, metabolic pathway were considered as enrichment, when P-value of metabolic pathway $<0.05$, metabolic pathway were considered as statistically significant enrichment.

\section{Results}

\section{Untargeted metabolic profiling of ovine and bovine serum at mid-lactation}

To detect the metabolic differences between cow, goat and sheep milk, an untargeted metabolomics analysis was performed and Human Metabolome Database (HMDB) was used to annotation. In total, 313 annotated metabolites from 1050 positive-ion feature and 173 annotated metabolites from 565 negative-ion feature were identified (Table S1). The results showed that the largest 
metabolic category was lipids and lipid-like molecules (188 metabolites), followed by organic acids and derivatives (98 metabolites) and organoheterocyclic compounds (55 metabolites) (Table S2).

In the positive-ion mode, the top 3 metabolites of ovine serum were Platelet-activating factor, Betaine and callystatin A and the top 3 metabolites of bovine serum were Hippuric acid, callystatin A and Platelet-activating factor. In the negative-ion mode, the top 3 metabolites of ovine serum were Oleic acid, Stearic acid and Ethyl myristate and the top 3 metabolites of bovine serum were Stearic acid, Ethyl myristate, Cholic acid. The results showed that high level of long-chain fatty acid at serum which could supply the formation of butterfat.

\section{Multivariate Statistical Analysis}

Principal Components Analysis (PCA) was used to determine the sample separation and aggregation between three milks. Each point on the PCA score graph represents a single sample. Aggregation of points indicates that the observed variables are highly similar, and discrete points represent significant differences (VIP $\geq 1$; ratio $\geq 2$ or ratio $\leq 1 / 2 ; q \leq 0.05)$ in the observed variables. In the positive-ion mode, the PCA scores illustrated that PC1 and PC2 were responsible for 53.25 and $17.79 \%$ of the variation, respectively (Figure 1A). In the negative-ion mode, the PCA scores revealed that PC1 and PC2 were responsible for 54.35 and $18.51 \%$ of the variation, respectively (Figure 1B). The results demonstrated that serum from different species had different metabolic characteristics.

To identify specific differences between groups, partial least squares discrimination analysis (PLS-DA) was used. Higher values for PLS-DA model parameters (R2 and Q2) denote greater reliability for the PLS-DA model. In the positive-ion mode, R2 of the PLS-DA model was 1.00, and Q2 was 0.99 (Figure 2A). Coincidentally, R2 of the PLS-DA model was 1.00 and Q2 was 0.99 in the negativeion mode (Figure 2B). The results indicated that both R2 and Q2 were high and subsequent analyses were credible.

\section{Differential metabolites analysis}

Next, we subjected the metabolomics data to univariate analysis of fold changes and T statistical testing to perform BenjaminiHochberg correction and obtain the P-value. This was combined with multivariate statistical analysis of the VIP obtained via PLSDA to screen for differential metabolites. Differential ions were defined as follows: VIP $\geq 1$; ratio $\geq 2$ or ratio $\leq 1 / 2 ; P \leq 0.05$. 269 and 143 metabolites were identified as differential metabolites in positive-ion and negative-ion modes, separately (Figure 3). In the positive-ion mode, 113 metabolites present higher level in ovine serum while 156 metabolites present higher level in bovine serum (Table S3). And 38 metabolites present higher level in ovine serum while 105 metabolites present higher level in bovine serum in the negative-ion mode (Table S3). The top 5 significant abundant metabolites of ovine serum were LAPPAOL C, 2-ETHYL-4,5DIMETHYLOXAZOLE, N-C18:0 Phytoceramide, (2S)-2-Amino-8-hydroxyoctanoic acid and carisoprodol (Table 1). The top 5 significant abundant metabolites of bovine serum were 4-Ethyl-2,6-dihydroxyphenyl hydrogen sulfate, (2R)-1-(Nonadecanoyloxy)-3(phosphonooxy)-2-propanyl docosanoate, Epinephrine, DG(16:1(9Z)/22:0/0:0) and tak-475 (Table 1).

Interestingly, much of metabolites which present higher level in ovine serum were associated with anti-microbico, antiviral or anticancer, such as Prunin, etravirine and Luteolin. While some of metabolites which present higher level in bovine serum were associated with contraception, such as gemeprost and Loxoprofen, which indicated that it may not suitable for pregnancy at this period. Notably, hernandezine, which is a novel AMPK activator, may play a role in the formation of lactoprotein of ovine milk.

\section{Pathway enrichment of differential abundant metabolites}

KEGG pathway enrichment showed that 18 and 10 functional pathways of differential metabolites were enriched at positive and negative ion mode, separately (Table 2). The most five enriched pathways of differential metabolites at positive-ion mode were Steroid hormone biosynthesis, Pathways in cancer, Prostate cancer, Purine metabolism and Oxidative phosphorylation (Table 2). The most five enriched pathways of differential metabolites at negative-ion mode were Carbohydrate digestion and absorption, Prion diseases, Insect hormone biosynthesis, Regulation of lipolysis in adipocytes and Aldosterone synthesis and secretion (Table 2). The results indicated that there may be different biological effects between two species serum.

\section{Discussion}


The blood metabolomics is one of an effective approach to discover the mechanism and prediction of livestock economic traits. The ovine milk owns higher percentages of lactoprotein and milk fat ${ }^{2}$. The blood serum metabolome of ovine and bovine at midlactation were analyzed to discover the mechanism. Among the metabolites, lappaol $\mathrm{C}$ and hernandezine were identified as high level at ovine serum. Lappaol $\mathrm{C}$ has antioxidant and antiaging properties, it may promote the $\mathrm{C}$. elegans longevity and stress resistance through a JNK-1-DAF-16 cascade ${ }^{12}$. Phospho-JNK play a role of phospho-AKT, and the phosphatidylinositol-3-kinase $(\mathrm{PI} 3 \mathrm{~K}) /$ Akt could activate the mTOR pathway ${ }^{13,14}$. Hernandezine is a noval activator of AMPK, which is one of the upstream targets of $\mathrm{mTOR}^{15-17} \square$ And the $\mathrm{mTOR}$ signaling is crucial for the synthesis of lactoprotein and milk fat ${ }^{18}$. In addition, the hernandezine also could inhibit the $\mathrm{Ca}^{2+}$ intake of calcium-depletion cells ${ }^{19,20}$, which may helpful for high calcium level of ovine milk. Based on these, we surmised that lappaol $\mathrm{C}$ and hernandezine may helpful for milk traits.

Thromboxane B2 is associated with arachidonic acid metabolism. Arachidonic acid and esterified arachidonate are ubiquitous components of every mammalian cell. This polyunsaturated fatty acid serves very important biochemical roles, including being the direct precursor of bioactive lipid mediators such as prostaglandin and leukotrienes ${ }^{21}$. High level of thromboxane B2 in ovine serum may be a marker of high polyunsaturated fatty acid in milk. Another research showed that arachidonic acid metabolites can promote angiogenesis in metastatic breast cancer ${ }^{22}$. Thus we surmise that it may contribute to angiogenesis of mammary gland during lactation. Flavin mononucleotide $(F M N)$ is a metabolite from vitamin B2. Without an adequate amount of vitamin B2, macronutrients like carbohydrates, fats, and proteins cannot be digested and maintain the body ${ }^{23}$. Vitamin B2 could improve the intake of protein and may helpful for milk protein biosynthesis. And the apoenzyme of lactate oxidase is specifically activated by FMN. FMN in serum may play a role for the biosynthesis of milk protein and fat.

\section{Conclusion}

In this study, the results showed that there are different metabolome profiles of ovine and bovine serum during lactation and distinct biological function. The metabolites of serum would affect the milk traits.

\section{Declarations}

\section{Author contributions}

Xiaohu Su contributed in design of experiments, analyzed the data and manuscript writing. Zhong Zheng obtained the samples, contributed to planning and design of the study. Liguo Zhang obtained the samples. Urhan Bai contributed to LC-MS analysis of samples and data collection. Guanghua Su contributed to experimental part and data analysis. Yunxi Wu obtained the samples. Guangpeng Li contributed to planning of the study and experiments. Li Zhang contributed to planning of the study and experiments, data collection and execution of experiments. All authors reviewed the manuscript.

\section{Ethics declarations}

Competing Interest

The authors declare no competing interests.

\section{Acknowledgements}

This work was funded by the Major Science and Technology project of Inner Mongolia Autonomous Region of China (309005173910), the Independent project of The State Key Laboratory of Reproductive Regulation and Breeding of Grassland Livestock of Inner Mongolia University of China (30500-518390205) and the Science and Technology Innovation Guidance project of Inner Mongolia Autonomous Region of China (30500-5173203). We owe many thanks to Mengtianran Dairy Co. Ltd. (Ulanqab, Inner Mongolia autonomous region, China) for the supply of place and animals. We owe many thanks to Novogene Co. LTD. (Beijing, China) for the metabolomics profiles processing.

\section{References}


1. Punia, H. et al. Identification and Detection of Bioactive Peptides in Milk and Dairy Products: Remarks about Agro-Foods. Molecules (Basel, Switzerland).25, https://doi.org/10.3390/molecules25153328 (2020).

2. Nguyen, H. T. H., Afsar, S. \& Day, L. Differences in the microstructure and rheological properties of low-fat yoghurts from goat, sheep and cow milk. Food research international (Ottawa, Ont.).108, 423-429 https://doi.org/10.1016/j.foodres.2018.03.040 (2018).

3. Goldansaz, S. A. et al. Livestock metabolomics and the livestock metabolome: A systematic review. PloS one.12, e0177675 https://doi.org/10.1371/journal.pone.0177675 (2017).

4. Foroutan, A., Fitzsimmons, C., Mandal, R., Berjanskii, M. V. \& Wishart, D. S. Serum Metabolite Biomarkers for Predicting Residual Feed Intake (RFI) of Young Angus Bulls. Metabolites.10, https://doi.org/10.3390/metabo10120491 (2020).

5. Funeshima, N. et al. Metabolomic profiles of plasma and uterine luminal fluids from healthy and repeat breeder Holstein cows. BMC veterinary research.17, https://doi.org/10.1186/s12917-021-02755-7 (2021).

6. Santos, A. et al. Liver transcriptomic and plasma metabolomic profiles of fattening lambs are modified by feed restriction during the suckling period. Journal of animal science.96, 1495-1507 https://doi.org/10.1093/jas/sky029 (2018).

7. Wang, B. et al. Arteriovenous blood metabolomics: An efficient method to determine the key metabolic pathway for milk synthesis in the intra-mammary gland. Scientific reports.8, 5598 https://doi.org/10.1038/s41598-018-23953-8 (2018).

8. Tian, H. et al. Integrated Metabolomics Study of the Milk of Heat-stressed Lactating Dairy Cows. Scientific reports.6, 24208 https://doi.org/10.1038/srep24208 (2016).

9. Ilves, A. et al. Alterations in milk and blood metabolomes during the first months of lactation in dairy cows. Journal of dairy science.95, 5788-5797 https://doi.org/10.3168/jds.2012-5617 (2012).

10. Wu, X. et al. Serum metabolome profiling revealed potential biomarkers for milk protein yield in dairy cows. Journal of proteomics.184, 54-61 https://doi.org/10.1016/j.jprot.2018.06.005 (2018).

11. Wang, H., Ding, J., Ding, S. \& Chang, Y. Metabolomic changes and polyunsaturated fatty acid biosynthesis during gonadal growth and development in the sea urchin Strongylocentrotus intermedius. Comparative biochemistry and physiology. Part $D$, Genomics \& proteomics.32, 100611 https://doi.org/10.1016/j.cbd.2019.100611 (2019).

12. Su, S. \& Wink, M. J. P. Natural lignans from Arctium lappa as antiaging agents in Caenorhabditis elegans. 117,340-350(2015).

13. Zhou, H., Zhang, Y., Chen, Q. \& Lin, Y. AKT and JNK Signaling Pathways Increase the Metastatic Potential of Colorectal Cancer Cells by Altering Transgelin Expression. Digestive diseases and sciences.61, 1091-1097 https://doi.org/10.1007/s10620-0153985-1 (2016).

14. Porta, C., Paglino, C. \& Mosca, A. Targeting PI3K/Akt/mTOR Signaling in Cancer. Frontiers in oncology.4, 64 https://doi.org/10.3389/fonc.2014.00064 (2014).

15. Li, P., Li, X., Wu, Y., Li, M. \& Wang, X. A novel AMPK activator hernandezine inhibits LPS-induced TNFa production. Oncotarget.8, 67218-67226 https://doi.org/10.18632/oncotarget.18365 (2017).

16. Song, Y. et al. Determination of a novel anticancer AMPK activator hernandezine in rat plasma and tissues with a validated UHPLC-MS/MS method: Application to pharmacokinetics and tissue distribution study. Journal of pharmaceutical and biomedical analysis.141, 132-139 https://doi.org/10.1016/j.jpba.2017.03.038 (2017).

17. Xu, J., Ji, J. \& Yan, X. H. Cross-talk between AMPK and mTOR in regulating energy balance. Critical reviews in food science and nutrition.52, 373-381 https://doi.org/10.1080/10408398.2010.500245 (2012).

18. Wang, Y. et al. Melatonin suppresses milk fat synthesis by inhibiting the mTOR signaling pathway via the MT1 receptor in bovine mammary epithelial cells. Journal of pineal research.67, e12593 https://doi.org/10.1111/jpi.12593 (2019).

19. Imoto, K. et al. Inhibitory effects of tetrandrine and hernandezine on $\mathrm{Ca} 2+$ mobilization in rat glioma $\mathrm{C} 6$ cells. Research communications in molecular pathology and pharmacology.95, 129-146 (1997).

20. Low, A. M. et al. Plant alkaloids, tetrandrine and hernandezine, inhibit calcium-depletion stimulated calcium entry in human and bovine endothelial cells. Life sciences.58, 2327-2335 https://doi.org/10.1016/0024-3205(96)00233-0 (1996).

21. Martin, S. A., Brash, A. R. \& Murphy, R. C. The discovery and early structural studies of arachidonic acid. Journal of lipid research.57, 1126-1132 https://doi.org/10.1194/jlr.R068072 (2016). 
22. Borin, T. F., Angara, K., Rashid, M. H., Achyut, B. R. \& Arbab, A. S. Arachidonic Acid Metabolite as a Novel Therapeutic Target in Breast Cancer Metastasis. International journal of molecular sciences.18, https://doi.org/10.3390/ijms18122661 (2017).

23. Mahabadi, N., Bhusal, A. \& Banks, S. W. in StatPearls (StatPearls Publishing Copyright @ 2020, StatPearls Publishing LLC 2020).

\section{Tables}

Table 1 The top 5 significant differential abundant metabolites of blood serum during lactation of ovine and bovine.

\begin{tabular}{|c|c|c|c|c|c|c|c|c|c|}
\hline Name_des & Formula & $\begin{array}{l}\text { Molecular } \\
\text { Weight }\end{array}$ & $\begin{array}{l}\text { Ovine } \\
\text { average }\end{array}$ & $\begin{array}{l}\text { Bovine } \\
\text { average }\end{array}$ & $\begin{array}{l}\text { Fold } \\
\text { Change }\end{array}$ & $\begin{array}{l}P \\
\text { value }\end{array}$ & $\mathrm{ROC}$ & VIP & Up.Down \\
\hline LAPPAOL C & $\begin{array}{l}\text { C30 } \\
\text { H34 } \\
\text { O10 }\end{array}$ & 554.21 & 912086.22 & 3435.83 & 265.463 & $\begin{array}{l}1.67 \mathrm{E}- \\
09\end{array}$ & 1.00 & 4.78 & up \\
\hline $\begin{array}{l}\text { 2-ETHYL-4,5- } \\
\text { DIMETHYLOXAZOLE }\end{array}$ & $\begin{array}{l}\text { C7 H11 } \\
\text { NO }\end{array}$ & 125.08 & 6457567.62 & 34758.38 & 185.785 & $\begin{array}{l}3.53 \mathrm{E}- \\
07\end{array}$ & 1.00 & 4.36 & up \\
\hline $\begin{array}{l}\text { N-C18:0 } \\
\text { Phytoceramide }\end{array}$ & $\begin{array}{l}\mathrm{C} 36 \\
\mathrm{H} 73 \mathrm{~N} \\
\mathrm{O} 4\end{array}$ & 583.55 & 460466.51 & 2500.33 & 184.162 & $\begin{array}{l}4.61 \mathrm{E}- \\
08\end{array}$ & 1.00 & 4.52 & up \\
\hline $\begin{array}{l}\text { (2S)-2-Amino-8- } \\
\text { hydroxyoctanoic acid }\end{array}$ & $\begin{array}{l}\mathrm{C} 8 \mathrm{H} 17 \\
\mathrm{~N} \mathrm{O} 3\end{array}$ & 175.12 & 6461861.25 & 35733.12 & 180.837 & $\begin{array}{l}5.62 \mathrm{E}- \\
07\end{array}$ & 1.00 & 4.33 & up \\
\hline carisoprodol & $\begin{array}{l}\mathrm{C} 12 \\
\mathrm{H} 24 \mathrm{~N} 2 \\
\mathrm{O} 4\end{array}$ & 260.17 & 332164.28 & 6878.27 & 48.292 & $\begin{array}{l}2.64 \mathrm{E}- \\
05\end{array}$ & 1.00 & 3.09 & up \\
\hline $\begin{array}{l}\text { 4-Ethyl-2,6- } \\
\text { dihydroxyphenyl } \\
\text { hydrogen sulfate }\end{array}$ & $\begin{array}{l}\text { C8 H10 } \\
\text { O6 S }\end{array}$ & 234.02 & 1226.86 & 105015.18 & 0.012 & $\begin{array}{l}2.58 \mathrm{E}- \\
03\end{array}$ & 1.00 & 2.75 & down \\
\hline $\begin{array}{l}\text { (2R)-1- } \\
\text { (Nonadecanoyloxy)-3- } \\
\text { (phosphonooxy)-2- } \\
\text { propanyl docosanoate }\end{array}$ & $\begin{array}{l}\mathrm{C} 44 \\
\mathrm{H} 87 \mathrm{O} 8 \\
\mathrm{P}\end{array}$ & 774.62 & 2960.50 & 288444.10 & 0.010 & $\begin{array}{l}1.12 \mathrm{E}- \\
08\end{array}$ & 1.00 & 3.96 & down \\
\hline Epinephrine & $\begin{array}{l}\mathrm{C} 9 \mathrm{H} 13 \\
\text { N O3 }\end{array}$ & 183.09 & 1911.49 & 251523.56 & 0.008 & $\begin{array}{l}1.33 \mathrm{E}- \\
04\end{array}$ & 1.00 & 3.44 & down \\
\hline DG(16:1(9Z)/22:0/0:0) & $\begin{array}{l}\text { C41 } \\
\text { H78 } 05\end{array}$ & 650.58 & 1534.23 & 300351.92 & 0.005 & $\begin{array}{l}3.02 \mathrm{E}- \\
04\end{array}$ & 1.00 & 4.03 & down \\
\hline tak-475 & $\begin{array}{l}\mathrm{C} 33 \\
\mathrm{H} 41 \mathrm{Cl} \\
\mathrm{N} 2 \mathrm{O}\end{array}$ & 644.25 & 1242.80 & 342837.71 & 0.004 & $\begin{array}{l}2.27 \mathrm{E}- \\
06\end{array}$ & 1.00 & 4.64 & down \\
\hline
\end{tabular}

Table 2 KEGG enrichment of significant differential abundant metabolites of blood serum during lactation of ovine and bovine. 


\begin{tabular}{|c|c|c|c|}
\hline & MapTitle & $\begin{array}{l}\mathrm{P} \\
\text { value }\end{array}$ & Metabolites \\
\hline \multirow[t]{18}{*}{$\begin{array}{l}\text { Positive ion } \\
\text { mode }\end{array}$} & Steroid hormone biosynthesis & 0.0027 & $\begin{array}{l}\text { Androstanolone, Testosterone, tetrahydrocortisol, } \\
\text { Cortisone }\end{array}$ \\
\hline & Pathways in cancer & 0.0043 & Androstanolone, Testosterone, Cortisone \\
\hline & Prostate cancer & 0.0043 & Androstanolone, Testosterone, Cortisone \\
\hline & Purine metabolism & 0.0757 & Xanthine \\
\hline & Oxidative phosphorylation & 0.1728 & Flavin mononucleotide \\
\hline & Caffeine metabolism & 0.1728 & Xanthine \\
\hline & Arachidonic acid metabolism & 0.1728 & Thromboxane B2 \\
\hline & Endocrine resistance & 0.1728 & Testosterone \\
\hline & Serotonergic synapse & 0.1728 & Thromboxane B2 \\
\hline & Ovarian steroidogenesis & 0.1728 & Testosterone \\
\hline & Aldosterone-regulated sodium reabsorption & 0.1728 & Cortisone \\
\hline & alpha-Linolenic acid metabolism & 0.3176 & Jasmonic acid \\
\hline & Insect hormone biosynthesis & 0.3176 & Juvenile hormone III \\
\hline & Riboflavin metabolism & 0.4385 & Flavin mononucleotide \\
\hline & $\begin{array}{l}\text { Neomycin, kanamycin and gentamicin } \\
\text { biosynthesis }\end{array}$ & 0.5393 & Paromamine \\
\hline & Porphyrin and chlorophyll metabolism & 0.5393 & pyropheophorbide a \\
\hline & Bile secretion & 0.6500 & Thromboxane B2, Aspirin \\
\hline & Vitamin digestion and absorption & 1.0000 & Flavin mononucleotide \\
\hline \multirow{10}{*}{$\begin{array}{l}\text { Negative ion } \\
\text { mode }\end{array}$} & Carbohydrate digestion and absorption & 0.1277 & Sucralose \\
\hline & Prion diseases & 0.1277 & Corticosterone \\
\hline & Insect hormone biosynthesis & 0.2414 & Ecdysterone \\
\hline & Regulation of lipolysis in adipocytes & 0.2414 & Corticosterone \\
\hline & Aldosterone synthesis and secretion & 0.2414 & Corticosterone \\
\hline & Steroid hormone biosynthesis & 0.3426 & Corticosterone \\
\hline & Phenylalanine metabolism & 0.3426 & Salicylic acid \\
\hline & Biosynthesis of unsaturated fatty acids & 1.0000 & Nervonic acid \\
\hline & Metabolic pathways & 1.0000 & Corticosterone, Luteolin, Salicylic acid \\
\hline & Bile secretion & 1.0000 & Salicylic acid \\
\hline
\end{tabular}

Figures 


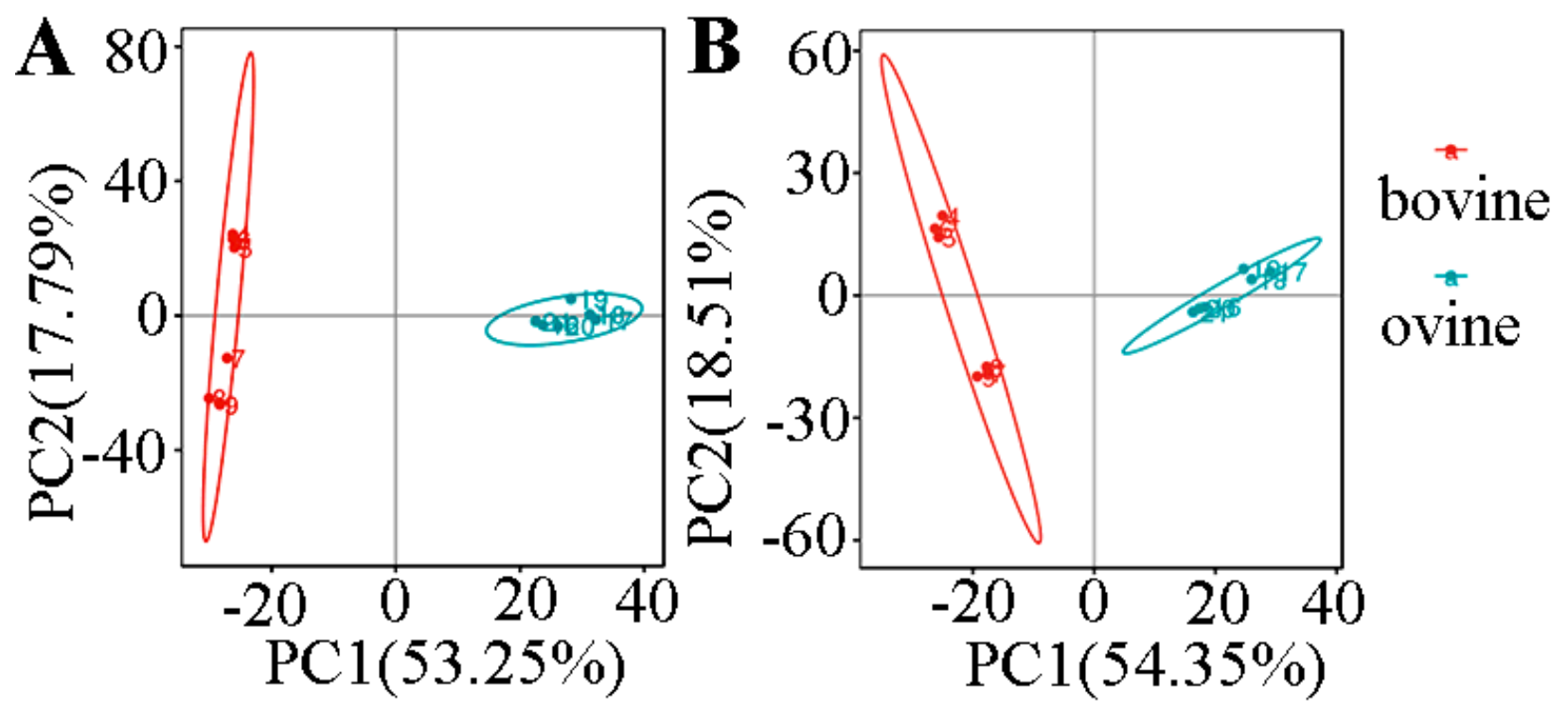

Figure 1

Principal components analysis (PCA) scores plot of metabolites identified in blood serum during lactation of ovine and bovine. A: positive-ion mode; B: negative-ion mode.

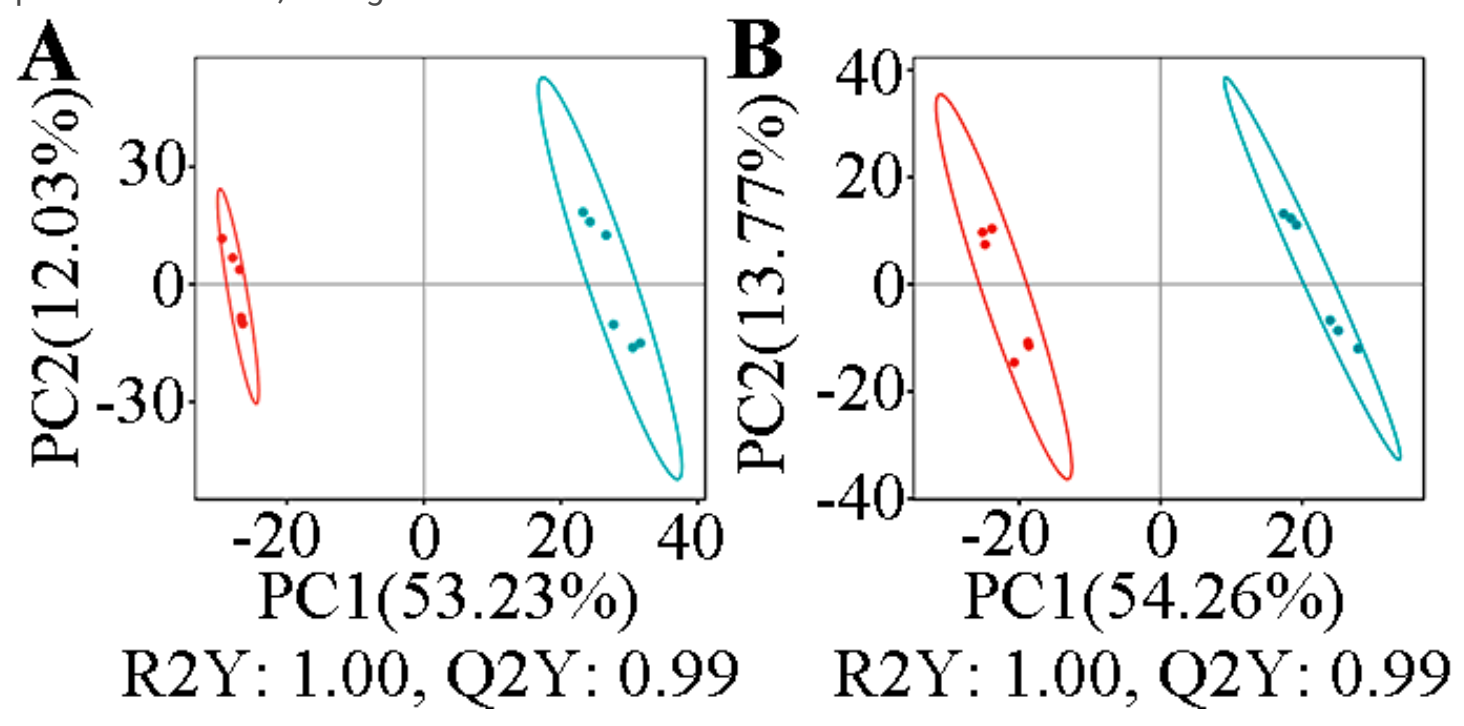

Figure 2

Partial least squares discrimination analysis (PLS-DA) scores plot of metabolites identified in blood serum during lactation of ovine and bovine. A: positive-ion mode; B: negative-ion mode. 


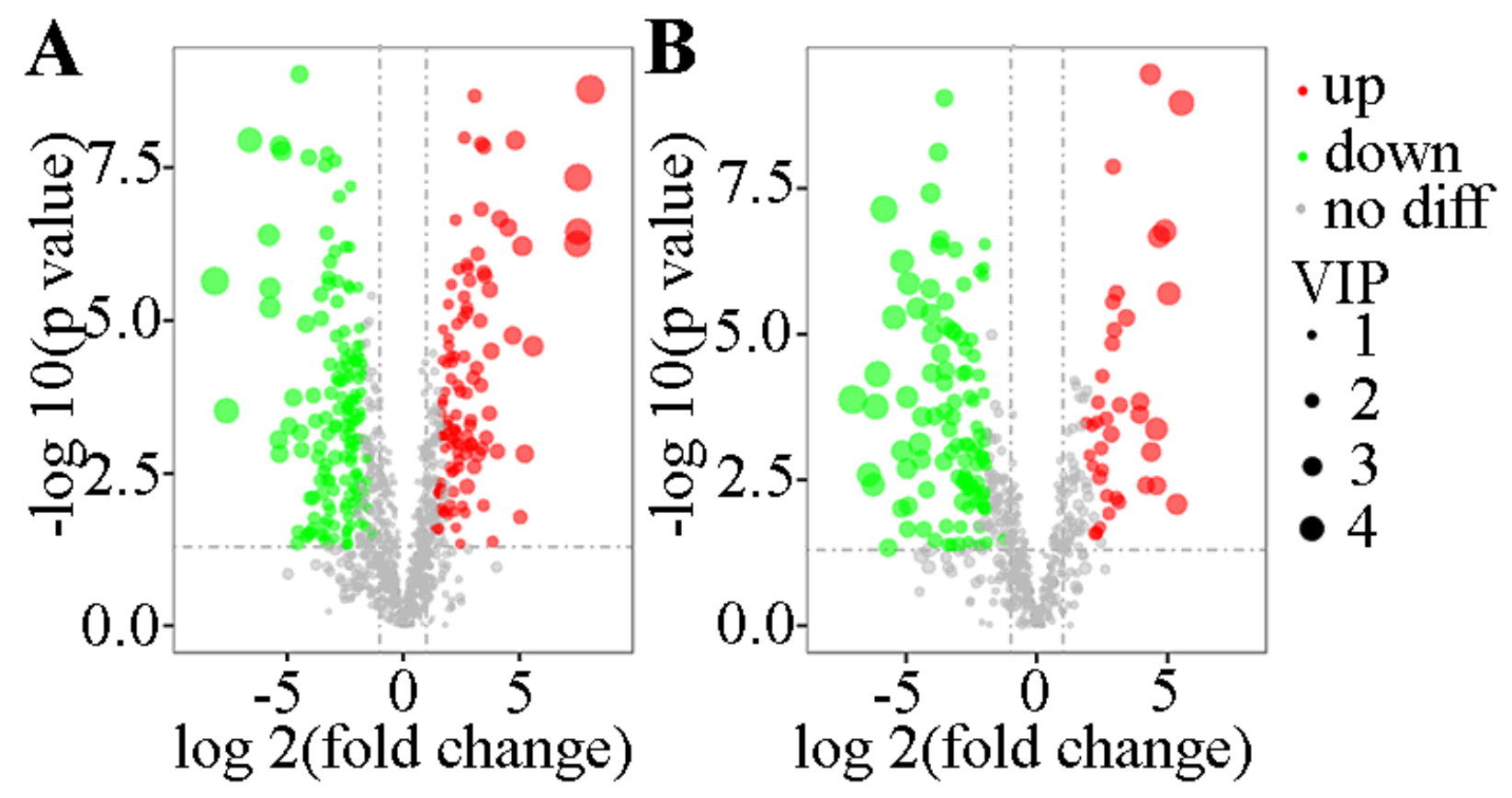

Figure 3

Volcano plot of metabolites identified in blood serum during lactation of ovine and bovine. A: positive-ion mode; B: negative-ion mode.

\section{Supplementary Files}

This is a list of supplementary files associated with this preprint. Click to download.

- Tables1.xlsx

- Tables2.xlsx

- TableS3.xls 